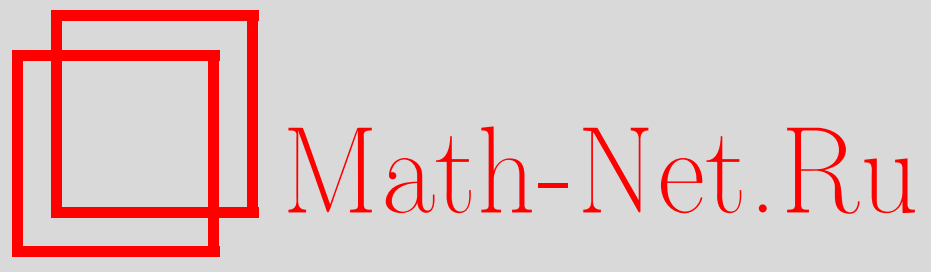

Е. М. Суханова, Многомерные знаковые и ранговые тесты независимости, УМН, 2008, том 63, выпуск 5, 199200

DOI: https://doi.org/10.4213/rm9226

Использование Общероссийского математического портала Math-Net.Ru подразумевает, что вы прочитали и согласны с пользовательским соглашением http: //www . mathnet.ru/rus/agreement

Параметры загрузки:

IP: 18.234 .197 .8

26 апреля 2023 г., $12: 35: 59$

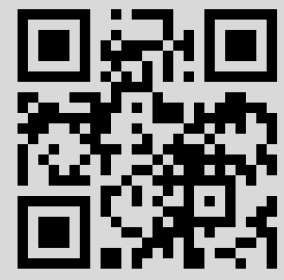




\section{Многомерные знаковые и ранговые тесты независимости}

\section{Е. М. Суханова}

Введение. В данной работе предложены новые матричнозначные корреляционные меры, обобщающие на многомерный случай хорошо известные непараметрические коэффициенты корреляции Спирмена (1904), Кендалла (1938) и Бломквиста (1950). В основе трех новых определений лежит понятие матричной корреляции, недавно появившееся в литературе [1]. Пусть $\mathbf{x}=\left(x_{1}, \ldots, x_{n}\right)$ и $\mathbf{y}=\left(y_{1}, \ldots, y_{n}\right)$ суть $n$ независимых реализаций случайных векторов $x \in \mathbb{R}^{p}, y \in \mathbb{R}^{q}$ соответственно. Обозначим выборочные матрицы ковариаций $\mathbf{s}_{11}:=\operatorname{ave}_{i}\left(x_{i}-\bar{x}\right)\left(x_{i}-\bar{x}\right)^{\prime}, \mathbf{s}_{22}:=$ $\operatorname{ave}_{i}\left(y_{i}-\bar{y}\right)\left(y_{i}-\bar{y}\right)^{\prime}$ и $\mathbf{s}_{12}:=\operatorname{ave}_{i}\left(x_{i}-\bar{x}\right)\left(y_{i}-\bar{y}\right)^{\prime}$. Матричной корреляицей $\mathbf{x}$ и $\mathbf{y}$ называется

$$
\mathbf{r}=\mathbf{r}(\mathbf{x}, \mathbf{y})=\mathbf{s}_{11}^{-1 / 2} \mathbf{s}_{12} \mathbf{s}_{22}^{-1 / 2} \text {. }
$$

Непараметрические матричные корреляции получаются из определения (1) заменой наблюдений на их многомерные знаки, ранги или на знаки попарных разностей. Здесь мы будем использовать понятия аффинно-эквивариантного многомерного знака и ранга, основанные на медиане Ойа; см., например, [2].

Аффинно-эквивариантные ранги и знаки. Пусть $\mathbf{x}=\left(x_{1}, \ldots, x_{n}\right)$ - выборка из $p$-мерного распределения с конечными первыми моментами. Положим $I=$ $\left\{i_{1}, \ldots, i_{p}\right\}, \quad J=\left\{i_{1}, \ldots, i_{p-1}\right\}$ для упорядоченных наборов индексов $i_{1}<\cdots<$ $i_{p-1}<i_{p}$; будем писать $\mathbf{x}_{I}=\left\{x_{i_{1}}, \ldots, x_{i_{p}}\right\}$ и аналогично $\mathbf{x}_{J}$. Аффинно-эквивариантная медиана Ойа $\widehat{\mu}_{\mathbf{x}}(1983)$ минимизирует целевую функцию $V(x ; \mathbf{x})=\operatorname{ave}_{I} V_{p}\left(x, \mathbf{x}_{I}\right)$, где $V_{p}\left(x, \mathbf{x}_{I}\right) / p$ ! - это абсолютный объем $p$-мерного симплекса с вершинами $x$ и $\mathbf{x}_{I}$.

Аффинно-эквивариантное расстояние между $x$ и началом координат может быть определено как $V_{0}(x ; \mathbf{x})=\operatorname{ave}_{J} V_{p}\left(x, \mathbf{x}_{J}, 0\right)$. Многомерными ранговой и знаковой функциями называются соответственно градиенты

$$
R(x ; \mathbf{x})=\nabla_{x} V(x ; \mathbf{x}), \quad S(x ; \mathbf{x})=\nabla_{x} V_{0}(x ; \mathbf{x}) .
$$

Обозначим ранги наблюдений $R_{i \mathbf{x}}=R\left(x_{i} ; \mathbf{x}\right)$. Для вычисления многомерных знаков $\widehat{S}_{i}$ наблюдений необходимо предварительно центрировать выборку с помощью медианы Ойа: $\widehat{S}_{i \mathbf{x}}=S\left(x_{i}-\widehat{\mu}_{\mathbf{x}} ; \mathbf{x}-\widehat{\mu}_{\mathbf{x}}\right)$.

Также мы будем рассматривать знаки попарных разностей наблюдений $x_{i j}=$ $x_{i}-x_{j}, \quad 1 \leqslant i<j \leqslant n$. Совокупность $\ddot{\mathbf{x}}:=\left\{x_{i j}\right\}$ уже центрирована, поэтому используется непосредственно знаковая функция (2): $S_{i j \mathbf{x}}=S\left(x_{i j} ; \ddot{\mathbf{x}}\right)$.

Новые тестовые статистики и их предельные распределения. Пусть дана выборка $\left\{\left(x_{i}^{\prime}, y_{i}^{\prime}\right)^{\prime}\right\}_{i=1}^{n}$ из симметричных $p$ - и $q$-мерных распределений с конечными вторыми моментами. Мы хотим проверить нулевую гипотезу $H_{0}: x_{i}$ и $y_{i}$ независимы.

Матричными аналогами коэффициентов корреляции Спирмена, Кендалла и Бломквиста (см., например, [3; разд. 4.6]) будем называть соответственно

$$
\begin{aligned}
\mathbf{r}_{S} & =\left(\operatorname{ave}_{i} R_{i \mathbf{x}} R_{i \mathbf{x}}^{T}\right)^{-1 / 2} \operatorname{ave}_{i} R_{i \mathbf{x}} R_{i \mathbf{y}}^{T}\left(\operatorname{ave}_{i} R_{i \mathbf{y}} R_{i \mathbf{y}}^{T}\right)^{-1 / 2}, \\
\mathbf{r}_{K} & =\left(\operatorname{ave}_{i, j, k} S_{i j \mathbf{x}} S_{i k \mathbf{x}}^{T}\right)^{-1 / 2} \operatorname{ave}_{i<j} S_{i j \mathbf{x}} S_{i j \mathbf{y}}^{T}\left(\operatorname{ave}_{i, j, k} S_{i j \mathbf{y}} S_{i k \mathbf{y}}^{T}\right)^{-1 / 2}, \\
\mathbf{r}_{Q} & =\left(\operatorname{ave}_{i} \widehat{S}_{i \mathbf{x}} \widehat{S}_{i \mathbf{x}}^{T}\right)^{-1 / 2} \operatorname{ave}_{i} \widehat{S}_{i \mathbf{x}} \widehat{S}_{i \mathbf{y}}^{T}\left(\operatorname{ave}_{i} \widehat{S}_{i \mathbf{y}} \widehat{S}_{i \mathbf{y}}^{T}\right)^{-1 / 2} .
\end{aligned}
$$

ЛЕмма 1. Сингулярные значения матрии $\mathbf{r}_{S}, \mathbf{r}_{K} u \mathbf{r}_{Q}$ инварианты относительно невырожденных аффинных преобразований $\left\{x_{i} \rightarrow A_{1} x_{i}+b_{1}\right\},\left\{y_{i} \rightarrow A_{2} y_{i}+b_{2}\right\}$.

Работа выполнена при поддержке РФФИ (проект № 06-01-00454). 
Новыми тестовыми статистиками для проверки гипотезы независимости $H_{0}$ будут служить нормы матричных корреляций $r_{S}=\left\|\mathbf{r}_{S}\right\|, r_{K}=\left\|\mathbf{r}_{K}\right\|, r_{Q}=\left\|\mathbf{r}_{Q}\right\|$, где $\|\mathbf{r}\|^{2}=\operatorname{Tr}\left(\mathbf{r r}^{\prime}\right)$. Из леммы 1 немедленно получаем аффинную инвариантность предложенных тестов. Поскольку $\mathbf{r}_{Q}$ использует медианы Ойа $\widehat{\mu}_{\mathbf{x}}, \widehat{\mu}_{\mathbf{y}}$, то требуется, чтобы они были $\sqrt{n}$-состоятельными оценками центров распределений (см. условия регулярности, например, в [4].

Теорема 1. При $H_{0}$ асимптотическое распределение $n r_{S}^{2}, n r_{K}^{2} / 4, n r_{Q}^{2}$ есть $\chi_{p q}^{2}$.

Предельное распределение при близких альтернативах получено для достаточно широкого класса эллиптических распределений. Поскольку тесты аффинно-инвариантны, можно ограничиться изучением сферического случая. Мы примем следующую модель: пусть $\left\{x_{i}^{*}\right\}$ и $\left\{y_{i}^{*}\right\}$ независимы и имеют стандартые сферические распределения $F$ и $G$; последовательность альтернатив имеет вид

$$
H_{n}: \quad\left(\begin{array}{l}
x_{i} \\
y_{i}
\end{array}\right)=\left(\begin{array}{cc}
(1-\Delta) I_{p} & \Delta M_{1} \\
\Delta M_{2} & (1-\Delta) I_{q}
\end{array}\right)\left(\begin{array}{l}
x_{i}^{*} \\
y_{i}^{*}
\end{array}\right), \quad \text { где } \Delta=\frac{\delta}{\sqrt{n}} .
$$

Для стандартного сферического распределения $x_{i}^{*} \sim F$ теоретическая ранговая функция имеет простую структуру: $R(x ; F):=\mathbf{E} R\left(x ; \mathbf{x}^{*}\right)=\alpha_{F}(\|x\|) x /\|x\|$. Аналогичное представление справедливо также для пространственного ранга $R_{1}(x ; F):=$ $\mathbf{E}\left(x-x_{i}^{*}\right) /\left\|x-x_{i}^{*}\right\|=\beta_{F}(\|x\|) x /\|x\|$. Явный вид функций $\alpha_{F}(\|x\|), \beta_{F}(\|x\|)$ для многомерного нормального и $t$-распределения можно найти в [2], [5]. Пусть $\mathbf{E} \alpha_{F}(\|x\|)^{2}=a_{F}^{2}$, $\mathbf{E} \beta_{F}(\|x\|)^{2}=b_{F}^{2}$. Эти обозначения также используем для сферического $y_{i} \sim G$.

Теорема 2. Пустъ $\min (p, q)>1$. При гипотезе $H_{n}$ асимптотическое распределение $n r_{S}^{2}, n r_{K}^{2} / 4, n r_{Q}^{2}$ есть $\chi_{p q}^{2}(\mathfrak{m})$ с параметром нецентральности $\mathfrak{m}=$ $\delta^{2}\left\|m_{1} M_{1}+m_{2} M_{2}^{\prime}\right\|^{2} /(p q)$, где постоянные $m_{1}, m_{2}$ имеют вид

$$
\begin{aligned}
& \text { для } r_{S}: \quad m_{1}=\frac{1}{a_{F} a_{G}}\left[(p-1) \mathbf{E} \frac{\alpha_{F}\left(\left\|x_{1}^{*}\right\|\right)}{\left\|x_{1}^{*}\right\|}+\mathbf{E} \alpha_{F}^{\prime}\left(\left\|x_{1}^{*}\right\|\right)\right] \mathbf{E} \alpha_{G}\left(\left\|y_{1}^{*}\right\|\right)\left\|y_{1}^{*}\right\|, \\
& m_{2}=\frac{1}{a_{F} a_{G}}\left[(q-1) \mathbf{E} \frac{\alpha_{G}\left(\left\|y_{1}^{*}\right\|\right)}{\left\|y_{1}^{*}\right\|}+\mathbf{E} \alpha_{G}^{\prime}\left(\left\|y_{1}^{*}\right\|\right)\right] \mathbf{E} \alpha_{F}\left(\left\|x_{1}^{*}\right\|\right)\left\|x_{1}^{*}\right\| \\
& \text { для } r_{K}: \quad m_{1}=\frac{1}{2 b_{F} b_{G}}(p-1) \mathbf{E} \frac{1}{\left\|x_{12}\right\|} \mathbf{E}\left\|y_{12}\right\|, \quad m_{2}=\frac{1}{2 b_{F} b_{G}}(q-1) \mathbf{E}\left\|x_{12}\right\| \mathbf{E} \frac{1}{\left\|y_{12}\right\|} ; \\
& \text { для } r_{Q}: \quad m_{1}=(p-1) \mathbf{E} \frac{1}{\left\|x_{1}^{*}\right\|} \mathbf{E}\left\|y_{1}^{*}\right\|, \quad m_{2}=(q-1) \mathbf{E}\left\|x_{1}^{*}\right\| \mathbf{E} \frac{1}{\left\|y_{1}^{*}\right\|} .
\end{aligned}
$$

Здесъ $\alpha_{F}^{\prime}, \alpha_{G}^{\prime}$ обозначают производные (существование которых предполагается).

Автор выражает глубокую благодарность профессорам Ю. Н. Тюрину и Х. Ойа за полезные обсуждения и ценные замечания.

\section{Список литературы}

[1] Е. М. Суханова, Теория вероятн. и ее примен. (в печати). [2] E. Ollila, Sign and rank covariance matrices with applications to multivariate analysis, Dissertation, Univ. of Jyväskylä, 2002. [3] J. Hájek, Z. Šidák, P. K. Sen, Theory of rank tests, 2nd ed., Probab. Math. Statist., Academic Press, San Diego, SA, 1999. [4] A. Arcones, Z. Chen, E. Giné, Ann. Statist., 22:3 (1994), 1460-1477. [5] J. Möttönen, H. Oja, J. Tienari, Ann. Statist., 25:2 (1997), 542-552.

\section{Е. М. Суханова (Е. M. Sukhanova)}

Московский государственный университет им. М. В. Ломоносова

E-mail: suhanova_katya@mail.ru
Представлено А. В. Булинским Принято редколлегией 20.06.2008 